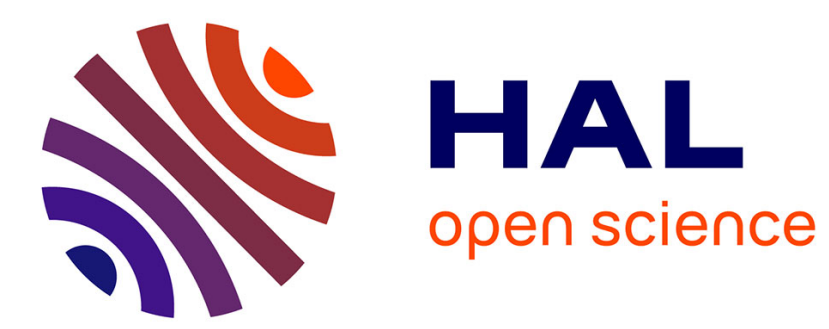

\title{
A REMARK ON RELATIVE GEOMETRIC INVARIANT THEORY FOR QUASI-PROJECTIVE VARIETIES
}

Alexander Schmitt

\section{> To cite this version:}

Alexander Schmitt. A REMARK ON RELATIVE GEOMETRIC INVARIANT THEORY FOR QUASI-PROJECTIVE VARIETIES. 2017. hal-01655234

\author{
HAL Id: hal-01655234 \\ https://hal.science/hal-01655234
}

Preprint submitted on 4 Dec 2017

HAL is a multi-disciplinary open access archive for the deposit and dissemination of scientific research documents, whether they are published or not. The documents may come from teaching and research institutions in France or abroad, or from public or private research centers.
L'archive ouverte pluridisciplinaire HAL, est destinée au dépôt et à la diffusion de documents scientifiques de niveau recherche, publiés ou non, émanant des établissements d'enseignement et de recherche français ou étrangers, des laboratoires publics ou privés. 


\title{
A REMARK ON RELATIVE GEOMETRIC INVARIANT THEORY FOR QUASI-PROJECTIVE VARIETIES
}

\author{
ALEXANDER H.W. SCHMITT
}

\begin{abstract}
Relative geometric invariant theory studies the behavior of semistable points under equivariant morphisms. More precisely, suppose $G$ is a complex reductive linear algebraic group, $X$ and $Y$ are quasi-projective varieties endowed with $G$-actions, $\varphi: X \longrightarrow Y$ is a $G$-equivariant projective morphism, the $G$-action on $Y$ is linearized in the ample line bundle $M$, and the $G$-action on $X$ is linearized in the $\varphi$-ample line bundle $L$. For any positive integer $n$, there is an induced linearization of the $G$-action on $X$ in the ample line bundle $L \otimes \varphi^{\star}\left(M^{\otimes n}\right)$. If $Y$ is projective and $n \gg 0$, the set of points in $X$ that are semistable with respect to this linearization is contained in the preimage under $\varphi$ of the set of points in $Y$ that are semistable with respect to the given linearization in $M$. The same statement is trivially also true, if $Y$ is affine. In this note, we show by means of an example that the statement does not hold for arbitrary quasi-projective varieties $Y$. This shows that a claim by $\mathrm{Hu}$ of the contrary is not true. Relative geometric invariant theory plays a role in the construction and study of degenerations of moduli spaces.
\end{abstract}

\section{INTRODUCTION}

Classical invariant theory deals with the ring of invariants of homogeneous forms of a given degree $d$ in a fixed set of variables $x_{0}, \ldots, x_{n}$. Gordan [1] managed to show that all invariants may be generated from a finite set of invariants in the case of binary forms, i.e., for $n=1$. His proof of is based on the so-called $\Omega$ process. A triumph of modern mathematics is Hilbert's proof for arbitrary $n$ [4]. In more modern language, we may look at a reductive linear algebraic group $G$, say, over the complex numbers, and a representation $\varrho: G \longrightarrow \mathrm{GL}(V)$ of $G$ on a finite dimensional complex vector space $V$. In the classical case, $G=\mathrm{SL}_{n+1}(\mathbb{C})$, $V=\mathbb{C}\left[x_{0}, \ldots, x_{n}\right]_{d}$, the space of homogeneous polynomials of degree $d$ in $x_{0}, \ldots, x_{n}$, and $\varrho$ comes from the action by linear substitutions of variables. The ring of regular functions on the affine algebraic variety $V$ is the symmetric algebra

$$
\operatorname{Sym}^{\star}\left(V^{\vee}\right)=\bigoplus_{d \in \mathbb{N}} \operatorname{Sym}^{d}\left(V^{\vee}\right)
$$

of the dual vector space $V^{\vee}$ to $V$. The group $G$ acts on this algebra by the formula

$$
\forall f \in \operatorname{Sym}^{\star}\left(V^{\vee}\right) \forall g \in G \forall v \in V: \quad(g \cdot f)(v):=f\left(\varrho\left(g^{-1}\right)(v)\right) .
$$

Hilbert's result asserts that the algebra

$$
\operatorname{Sym}^{\star}\left(V^{\vee}\right)^{G}:=\left\{f \in \operatorname{Sym}^{\star}\left(V^{\vee}\right) \mid \forall g \in G: g \cdot f=f\right\}
$$

is a finitely generated $\mathbb{C}$-algebra. His original proof is non-constructive. However, in [5], he conceived a beautiful approach for determining the ring of invariants. It is based on his notion of semistability and the criterion that is nowadays known as the Hilbert-Mumford criterion. Here, a point $v \in V$ is called a nullform, if all homogeneous invariant polynomials on $V$ vanish in $v$, i.e., for all $d \geq 0$ and $f \in \operatorname{Sym}^{d}\left(V^{\vee}\right)^{G}, f(v)=0$. The opposite of a nullform is a semistable point.

Mumford realized that Hilbert's theory may be used as a tool for solving classification problems in algebraic geometry. Let us start discussing this by looking at 
the action $\bar{\sigma}: G \times P(V) \longrightarrow P(V)$ of $G$ on the projective space $P(V)$ of lines in $V$ that is induced by $\varrho$. Since $\operatorname{Sym}^{\star}\left(V^{\vee}\right)^{G}$ is a finitely generated graded algebra, we may form the projective variety

$$
P(V) / / \varrho G:=\operatorname{Proj}\left(\operatorname{Sym}^{\star}\left(V^{\vee}\right)^{G}\right) .
$$

Define

$$
P(V)^{\mathrm{ss}}(\varrho):=\{[v] \in P(V) \mid v \text { is semistable }\} .
$$

It is a $G$-invariant open subset of $P(V)$. By definition, there is a $G$-invariant morphism

$$
\pi_{\varrho}: P(V)^{\mathrm{ss}}(\varrho) \longrightarrow P(V) / / \varrho G .
$$

It is part of Mumford's insight that this is a categorical quotient ([8], Theorem $1.10)$. If $G=\mathrm{SL}_{n+1}(\mathbb{C}), V=\mathbb{C}\left[x_{0}, \ldots, x_{n}\right]_{d}$, and $\varrho$ comes from the action by linear substitutions of variables, then $P(V)$ is the linear system of hypersurfaces of degree $d$ in $\mathbb{P}_{n}$ and $P(V) / / \varrho G$ is the moduli space of projective equivalence classes of these hypersurfaces.

Returning to arbitrary representations, we may, more generally, look at a $G$ invariant closed subvariety $X \hookrightarrow P(V)$, set $X^{\mathrm{ss}}(\varrho):=X \cap P(V)^{\mathrm{ss}}(\varrho)$, and define $X / / \varrho G$ as the image of $X^{\mathrm{ss}}(\varrho)$ under $\pi_{\varrho}$. Then, $X / / \varrho_{\varrho} G$ is a projective variety and

$$
\pi_{\varrho \mid X^{\mathrm{ss}}(\varrho)}: X^{\mathrm{ss}}(\varrho) \longrightarrow X / / \varrho G
$$

is a categorical quotient. As an example, suppose we are given a second representation $\tau: G \longrightarrow \mathrm{GL}(W)$. Then, $\varrho$ and $\tau$ induce an action $\bar{\alpha}: G \times P(V) \times P(W) \longrightarrow$ $P(V) \times P(W)$. For positive integers $l, m$, we may form the representation $\beta_{l, m}:=$ $\operatorname{Sym}^{l}(\varrho) \otimes \operatorname{Sym}^{m}(\tau): G \longrightarrow \operatorname{GL}\left(U_{l, m}\right), U_{l, m}:=\operatorname{Sym}^{l}(\varrho) \otimes \operatorname{Sym}^{m}(\tau)$. Then, we get the $G$-invariant closed Segre embedding

$$
\iota_{m, n}: P(V) \times P(W) \longleftrightarrow P\left(U_{l, m}\right) .
$$

The resulting open subset of semistable points in $P(V) \times P(W)$ depends only on the ratio $\eta:=m / l$, so we denote it by $(P(V) \times P(W))^{\mathrm{ss}}(\eta)$. It is part of the study of variation of quotients (see [12], Section 1.6) to investigate how $(P(V) \times P(W))^{\mathrm{ss}}(\eta)$ and the resulting categorical quotient vary with $\eta$. A basic observation is the following

Proposition. There is a rational number $\eta_{\infty}$, such that, for all parameters $\eta>\eta_{\infty}$,

$$
(P(V) \times P(W))^{\mathrm{ss}}(\eta) \subset \pi_{P(W)}^{-1}\left(P(W)^{\mathrm{ss}}(\tau)\right),
$$

$\pi_{P(W)}: P(V) \times P(W) \longrightarrow P(W)$ being the projection onto the second factor.

Proof. This is [10], Theorem 2.1, a). It also follows readily from [12], Proposition 1.7.3.1.

Now, one may be tempted to apply this observation to a $G$-invariant locally closed subvariety $Y \subset P(W)$, a $G$-invariant closed subvariety $X \hookrightarrow P(V) \times Y$, and the resulting $G$-equivariant projective morphism $\varphi: X \longrightarrow Y$. In fact, it immediately applies, if $Y$ is a closed subvariety. If $Y$ is affine, it works, too, and leads to an interesting new Hilbert-Mumford criterion (see [3], [13]). Hu ([6], Theorem 3.11 ) claims that it also works for arbitrary $Y$. The aim of this note is to show that Hu's assertion is, in fact, wrong, by giving a counter example. This is related to the fact that the Hilbert-Mumford criterion of King, Gulbrandsen/Halle/Hulek does not extend to arbitrary quasi-projective varieties (see [14]). Since problems in relative geometric invariant theory do occur in the construction of moduli spaces, such as the moduli space of semistable torsion free sheaves or Gieseker bundles over the moduli space $\overline{\mathcal{M}}_{g}$ of stable curves of genus $g$ ([9] and [11], respectively), and in studying degenerations of moduli spaces, such as Hilbert schemes relative to a 
semi-stable degeneration of curves ([3], Section 6.2), it seems worthwhile to acquire a deep understanding of this setting.

Notation. Given a finite dimensional complex vector space $V$, we write $P(V)$ for the projective space of lines in $V$ and $\mathbb{P}(V)$ for the projective space of hyperplanes in $V$. A line bundle $L$ will be thought of as a geometric object as in [2], Definition 11.5. If necessary, it will be identified with the invertible sheaf $\mathcal{L}$ with $L=\operatorname{Spec}\left(\operatorname{Sym}^{\star}\left(\mathcal{L}^{\vee}\right)\right)$, i.e., the sheaf of sections of $L$. We will work over the field of complex $\overline{\text { numbers. }}$

Acknowledgment. This research was supported by Priority Programme 1786 "Homotopy Theory and Algebraic Geometry" of the German Research Foundation DFG.

\section{LineARIZATIONS AND SEMISTABILITY}

Let $X$ be an algebraic variety, $G$ a reductive linear algebraic group, and $\bar{\sigma}: G \times$ $X \longrightarrow X$ an action of $G$ on $X$. Fix also a line bundle $p: L \longrightarrow X$. A linearization of $\bar{\sigma}$ in $L$ is an action $\sigma: G \times L \longrightarrow L$, such that

- $p$ is $G$-equivariant,

- the isomorphism $G \times L \longrightarrow \bar{\sigma}^{\star}(L)$ induced by $\sigma$ is an isomorphism of line bundles over $G \times X$.

A point $x \in X$ is $\sigma$-semistable, if there exist a positive integer $r$ and a $G$-invariant section $s \in H^{0}\left(X, L^{\otimes r}\right)$, such that the $G$-invariant open subset

$$
X_{s}:=\{x \in X \mid s(x) \neq 0\}
$$

is affine and contains $x$. The $\sigma$-semistable points form the $G$-invariant open subset $X^{\mathrm{ss}}(\sigma)$ of $X$. A point $x \in X$ is said to be $\sigma$-polystable, if it is $\sigma$-semistable and its orbit $G \cdot x$ is a closed subset of $X^{\mathrm{ss}}(\sigma)$. Finally, a point $x \in X$ is $\sigma$-stable, if it is $\sigma$-polystable and its stabilizer $G_{x}$ is a finite group. The set $X^{\mathrm{s}}(\sigma)$ of $\sigma$-stable points is also open and $G$-invariant.

Example 1.1. Let $\varrho: G \longrightarrow \mathrm{GL}(V)$ be a rational representation of $G$ on the finite dimensional complex vector space $V$. It induces an action $\bar{\sigma}: G \times P(V) \longrightarrow P(V)$ and a linearization of this action in the tautological line bundle $\mathcal{O}_{P(V)}(-1)$. We let $\sigma: G \times \mathcal{O}_{P(V)}(1) \longrightarrow \mathcal{O}_{P(V)}(1)$ be the induced linearization in the dual of the tautological line bundle. In the notation of the introduction, we have

$$
P(V)^{\mathrm{ss}}(\sigma)=P(V)^{\mathrm{ss}}(\varrho) .
$$

For later reference, we set $P(V)^{\mathrm{s}}(\varrho):=P(V)^{\mathrm{s}}(\sigma)$.

\section{The Hilbert-Mumford CRITERION}

Let $X$ be a projective algebraic variety, $G$ a reductive linear algebraic group, $\bar{\sigma}: G \times X \longrightarrow X$ an action of $G$ on $X, L$ a line bundle on $X$, and $\sigma: G \times L \longrightarrow L$ a linearization of $\bar{\sigma}$ in $L$. For a one parameter subgroup $\lambda: \mathbb{C}^{\star} \longrightarrow G$ and a point $x \in X$, we get the morphism

$$
\begin{aligned}
\mathbb{C}^{\star} & \longrightarrow X \\
z & \longmapsto \bar{\sigma}(\lambda(z), x) .
\end{aligned}
$$

It extends to the point $\infty \in \mathbb{P}_{1}$, and the value $x_{\infty} \in X$ of the extension at $\infty$ is a fix point for the $\mathbb{C}^{\star}$-action on $X$ induced by $\bar{\sigma}$ and $\lambda$. Via $\sigma, \mathbb{C}^{\star}$ acts on the fiber of $L$ over $x_{\infty}$, and there exists an integer $\gamma$, such that this action is of the form $(z, \ell) \longmapsto z^{\gamma} \cdot \ell$. We define

$$
\mu_{\sigma}(\lambda, x):=-\gamma
$$


Example 2.1. Let the situation be as in Example 1.1. For a one parameter subgroup $\lambda: \mathbb{C}^{\star} \longrightarrow G$, the representation $\varrho \circ \lambda$ of $\mathbb{C}^{\star}$ is diagonalizable, i.e., there exist integral weights $\gamma_{1}<\cdots<\gamma_{s}$, such that

$$
V_{i}:=\left\{v \in V \mid \forall z \in \mathbb{C}^{\star}:(\varrho \circ \lambda)(z)(v)=z^{\gamma_{i}} \cdot v\right\} \neq\{0\}, \quad i=1, \ldots, s,
$$

and

$$
V=V_{1} \oplus \cdots \oplus V_{s}
$$

For a non-zero vector $v=\left(v_{1}, \ldots, v_{s}\right) \in V$, we have

$$
\mu_{\sigma}(\lambda,[v])=\mu_{\varrho}(\lambda, v):=\max \left\{\gamma_{i} \mid v_{i} \neq 0, i=1, \ldots, s\right\} .
$$

The Hilbert-Mumford criterion ([8], Theorem 2.1) asserts that a point $x \in X$ is $\sigma$-(semi)stable if and only if the inequality

$$
\mu_{\sigma}(\lambda, x)(\geq) 0
$$

holds for every non-constant one parameter subgroup $\lambda: \mathbb{C}^{\star} \longrightarrow G$.

Example 2.2. Suppose $\varrho: G \longrightarrow \mathrm{GL}(V)$ and $\tau: G \longrightarrow \mathrm{GL}(W)$ are two finite dimensional rational representations of $G$. As noted in the introduction, they induce an action $\bar{\alpha}: G \times P(V) \times P(W) \longrightarrow P(V) \times P(W)$. The linearization of the $G$-action on $P(V)$ in $\mathcal{O}_{P(V)}(1)$ and of the $G$-action on $P(W)$ in $\mathcal{O}_{P(W)}(1)$ induce, for any two positive integers $l, m$, a linearization $\alpha_{l, m}$ of $\bar{\alpha}$ in

$$
\mathcal{O}_{P(V) \times P(W)}(l, m):=\pi_{P(V)}^{\star}\left(\mathcal{O}_{P(V)}(l)\right) \otimes \pi_{P(W)}^{\star}\left(\mathcal{O}_{P(W)}(m)\right) .
$$

Proposition. For any two positive integers $l, m$, we have

$$
P(V)^{\mathrm{s}}(\varrho) \times P(W)^{\mathrm{ss}}(\tau) \subset(P(V) \times P(W))^{\mathrm{s}}\left(\alpha_{l, m}\right) .
$$

Proof. Let $v \in V$ and $w \in W$ be non-zero vectors, such that $[v] \in P(V)^{\mathrm{s}}(\varrho)$ (see Example 1.1 for the notation) and $[w] \in P(W)^{\mathrm{ss}}(\tau)$. Let $\lambda: \mathbb{C}^{\star} \longrightarrow G$ be a nonconstant one parameter subgroup. By the Hilbert-Mumford criterion on $P(V)$ and $P(W)$, we have

$$
\mu_{\alpha_{l, m}}(\lambda,([v],[w]))=l \cdot \mu_{\varrho}(\lambda, v)+m \cdot \mu_{\tau}(\lambda, w)>m \cdot \mu_{\tau}(\lambda, w) \geq 0 .
$$

The Hilbert-Mumford criterion shows that the point $([v],[w])$ is $\alpha_{l, m}$-stable

\section{Semistability And immersions}

Mumford ([8], Chapter 1, §5) studied how the sets of stable and semistable points behave under certain kinds of morphisms. E.g., it is rather straightforward to check that the formation of the set of (semi)stable points is compatible with closed embeddings. However, for locally closed immersions, things are already quite delicate. Here, we will briefly discuss Mumford's results for some special locally closed immersions.

Let $X$ be a quasi-projective variety, $G$ a reductive linear algebraic group, $\bar{\sigma}: G \times$ $X \longrightarrow X$ an action of $G$ on $X, L$ an ample line bundle on $X$, and $\sigma: G \times L \longrightarrow L$ a linearization of $\bar{\sigma}$ in $L$. For a positive integer $r$, the space $H^{0}\left(X, L^{\otimes r}\right)$ may be infinite dimensional, but the action of $G$ on it is locally finite, i.e., every finite dimensional subspace $W \subset H^{0}\left(X, L^{\otimes r}\right)$ is contained in a finite dimensional $G$-invariant subspace $V \subset H^{0}\left(X, L^{\otimes r}\right)$. The group $G$ acts on $V$ via a rational representation. So, there are an induced action $\bar{\alpha}: G \times \mathbb{P}(V) \longrightarrow \mathbb{P}(V)$ and a linearization $\alpha: G \times \mathcal{O}_{\mathbb{P}(V)}(1) \longrightarrow \mathcal{O}_{\mathbb{P}(V)}(1)$ of the action in the tautological line bundle on $\mathbb{P}(V)$. The rational map

$$
\psi: X--\rightarrow \mathbb{P}(V)
$$

defined by the evaluation homomorphism ev: $V \otimes \mathcal{O}_{X} \longrightarrow L$ is $G$-equivariant. 
Proposition 3.1. There are a positive integer $r$ and a $G$-invariant finite dimensional $G$-invariant subspace $V \subset H^{0}\left(X, L^{\otimes r}\right)$, such that

o) The rational map $\psi$ defined above is a locally closed immersion.

i) A point $x \in X$ is $\sigma$-polystable if and only if $\psi(x)$ is $\alpha$-polystable.

ii) A point $x \in X$ is $\sigma$-semistable if and only if $\psi(x)$ is $\alpha$-semistable and

$$
\overline{G \cdot \psi(x)} \cap\left(\mathbb{P}(V)^{\mathrm{ss}}(\alpha)\right) \subset \psi(X) .
$$

Proof. [12], Theorem 1.4.3.7. (It is basically [8], Amplification 1.8.)

Remark 3.2. i) Since stable points are polystable points with finite stabilizer, Part i) is also true for stable points.

ii) As is apparent from the proof of the proposition, the same assertion is true for any $G$-invariant finite dimensional $G$-invariant subspace $V^{\prime} \subset H^{0}\left(X, L^{\otimes r}\right)$ which contains $V$.

iii) Assume that $P$ is a non-singular projective variety, $L$ is an ample line bundle on $P, X \subset P$ is an open subset, such that the complement $Z:=P \backslash X$ has codimension at least two in $P$, and $L_{X}$ is the restriction of $L$ to $X$. Then, by the algebraic Hartogs lemma ([2], Theorem 6.45),

$$
\forall n>0: \quad H^{0}\left(X, L_{X}^{\otimes n}\right)=H^{0}\left(P, L^{\otimes n}\right) .
$$

In particular, $H^{0}\left(X, L_{X}^{\otimes n}\right)$ is finite dimensional, for any $n>0$.

Now, suppose that $G$ is a reductive linear algebraic group, $\bar{\sigma}: G \times P \longrightarrow P$ is an action of $G$ on $P, \sigma: G \times L \longrightarrow L$ is a linearization of $\bar{\sigma}$ in $L$, and $X$ is $G$ invariant. Let $\bar{\sigma}_{X}: G \times X \longrightarrow X$ and $\sigma_{X}: G \times L_{X} \longrightarrow L_{X}$ be the induced action and linearization, respectively. By the previous remark, we may take $V=H^{0}\left(X, L_{X}^{\otimes r}\right)$ in the statement of the proposition. In addition, the first remark and Part i) of the proposition show

$$
X^{\mathrm{s}}\left(\sigma_{X}\right)=X \cap P^{\mathrm{s}}(\sigma)
$$

\section{A counterexample to a Claim by Hu}

Let $G$ be a connected reductive affine algebraic group, $V$ a finite dimensional complex vector space, and $\varrho: G \longrightarrow \mathrm{GL}(V)$ a representation of $G$ on $V$. We use the notation of Example 1.1. Pick a vector $w_{0} \in V \backslash\{0\}$, such that

- $\left[w_{0}\right] \in P(V)$ is $\sigma$-semistable but not $\sigma$-polystable,

- the orbit of $\left[w_{0}\right]$ is not dense.

The closure of the orbit of $\left[w_{0}\right]$ inside the set $P(V)^{\mathrm{ss}}(\sigma)$ of $\sigma$-semistable points contains a unique closed orbit $O$. We define $Z$ to be the closure of $O$ in $P(V)$ and $Y:=P(V) \backslash Z$. This is a $G$-invariant open subset of $P(V)$, so, it inherits an action $\bar{\sigma}_{Y}: G \times Y \longrightarrow Y$. Define $L_{Y}$ as the restriction of $\mathcal{O}_{P(V)}(1)$ to $Y$. The linearization $\sigma$ restricts to a linearization $\sigma_{Y}: G \times L_{Y} \longrightarrow L_{Y}$ of $\bar{\sigma}_{Y}$ in $L_{Y}$. We shall also assume

- $P(V)^{\mathrm{s}}(\sigma) \neq \varnothing$.

Example 4.1 (Binary quartics). Look at the action of the group $G:=\mathrm{SL}_{2}(\mathbb{C})$ on the vector space $V:=\mathbb{C}\left[x_{0}, x_{1}\right]_{4}$ of binary homogeneous polynomials of degree four discussed in the introduction. It is well-known (see, e.g., [12], Lemma 1.3.2.6) that a binary quartic form $f \in V \backslash\{0\}$ is

- semistable if and only if it has no zero of multiplicity three or four,

- stable if and only if it has only roots of multiplicity one, and

- polystable if and only if it has only roots of multiplicity one, or two roots of multiplicity two.

So, the set of stable points is non-empty, and the form $x_{0}^{2} \cdot x_{1}^{2}+x_{0} \cdot x_{1}^{3}$ is semistable and contains the polystable form $x_{0}^{2} \cdot x_{1}^{2}$ in the closure of its orbit. 
Proposition 4.2. The point $\left[w_{0}\right] \in Y \cap P(V)^{\mathrm{ss}}(\varrho)$ is not $\sigma_{Y}$-semistable.

Proof. We refer to [14], Theorem 3.2.

Now, we define $X:=P(V) \times Y$ and let $\varphi: X \longrightarrow Y$ be the projection onto the second factor. We use the notation from Example 2.2 for $\varrho=\tau$. For positive integers $l, m$, let $L_{l, m}$ be the restriction of $\mathcal{O}_{P(V) \times P(W)}(l, m)$ to $X$. Denote by $\bar{\beta}: G \times X \longrightarrow X$ the induced action and by $\beta_{l, m}: G \times L_{l, m} \longrightarrow L_{l, m}$ the linearization coming from $\alpha_{l, m}, l, m$ positive integers.

Proposition 4.3. For $l, m \in \mathbb{Z}_{>0}, P(V)^{\mathrm{s}}(\varrho) \times\left(Y \cap P(V)^{\mathrm{ss}}(\varrho)\right) \subset X^{\mathrm{s}}\left(\beta_{l, m}\right)$.

Proof. The proposition in Example 2.2 implies

$$
P(V)^{\mathrm{s}}(\varrho) \times\left(Y \cap P(V)^{\mathrm{ss}}(\varrho)\right) \subset(P(V) \times P(V))^{\mathrm{s}}\left(\alpha_{l, m}\right) .
$$

By Remark 3.2, iii),

$$
(P(V) \times P(V))^{\mathrm{s}}\left(\alpha_{l, m}\right) \cap X=X^{\mathrm{s}}\left(\beta_{l, m}\right) .
$$

These facts imply the claim.

An immediate consequence is the following.

Theorem 4.4. There do not exist positive integers $l, m$, such that

$$
X^{\mathrm{ss}}\left(\beta_{l, m}\right) \subset \varphi^{-1}\left(Y^{\mathrm{ss}}\left(\sigma_{Y}\right)\right) .
$$

In particular, [6], Theorem 3.11 (1), does not hold for the morphism $\varphi$.

Remark 4.5. The gap in Hu's proof is easy to spot. In the notation of [6], Lemma 3.10 is applied to the immersion $X \longrightarrow \mathbb{P}^{N}$ which is not a closed immersion, if $X$ is not projective.

\section{REFERENCES}

[1] P. Gordan, Beweis, dass jede Covariante und Invariante einer binären Form eine ganze Function mit numerischen Coefficienten einer endlichen Anzahl solcher Formen ist, J. reine angew. Math. 69 (1868), 323-54.

[2] U. Görtz, T. Wedhorn, Algebraic geometry I. Schemes with examples and exercises, Advanced Lectures in Mathematics, Vieweg + Teubner, Wiesbaden, 2010, viii+615 pp.

[3] M.G. Gulbrandsen, L.H. Halle, K. Hulek, A relative Hilbert-Mumford criterion, Manuscripta Math. 148 (2015), 283-301.

[4] D. Hilbert, Ueber die Theorie der algebraischen Formen, Math. Ann. 36 (1890), 473-534.

[5] D. Hilbert, Ueber die vollen Invariantensysteme, Math. Ann. 42 (1893), 313-73.

[6] Y. Hu, Relative geometric invariant theory and universal moduli spaces, Int. J. Math. 7 (1996), 151-81.

[7] A.D. King, Moduli of representations of finite-dimensional algebras, Quart. J. Math. Oxford Ser. (2) 45 (1994), 515-30.

[8] D. Mumford, J. Fogarty, F. Kirwan, Geometric invariant theory, third edition, Ergebnisse der Mathematik und ihrer Grenzgebiete (2), vol. 34, Springer-Verlag, Berlin, 1994, xiv +292 pp.

[9] R. Pandharipande, A compactification over $\bar{M}_{g}$ of the universal moduli space of slopesemistable vector bundles, J. Amer. Math. Soc. 9 (1996), 425-71.

[10] Z. Reichstein, Stability and equivariant maps, Invent. Math. 96 (1989), 349-83.

[11] A.H.W. Schmitt, The Hilbert compactification of the universal moduli space of semistable vector bundles over smooth curves, J. Differential Geom. 66 (2004), 169-209.

[12] A.H.W. Schmitt, Geometric invariant theory and decorated principal bundles, Zurich Lectures in Advanced Mathematics, European Mathematical Society (EMS), Zürich, 2008, viii+389 pp.

[13] A.H.W. Schmitt, Semistability and instability in products and applications, String-Math 2014, 201-14, Proc. Sympos. Pure Math., vol. 93, Amer. Math. Soc., Providence, RI, 2016.

[14] A.H.W. Schmitt, Around the Hilbert-Mumford criterion, preprint, 2017, 4 pp.

Freie Universität Berlin, Institut für Mathematik, Arnimallee 3, D-14195 Berlin, GERMANY

E-mail address: alexander.schmitt@fu-berlin.de 